\title{
Conocimiento y uso de las plantas medicinales en la zona metropolitana de Guadalajara*
}

\author{
Javier E. García de Alba García, Blanca C. Ramírez Hernández, \\ Gilberto Robles Arellano, Julia Zañudo Hernández, \\ Ana L. Salcedo Rocha y Javier E. García de Alba Verduzco
}

Se destaca el conocimiento popular de "herbolarios, hierberos, yerberos o yerbateros" sobre las plantas medicinales utilizadas en mercados de la Zona Metropolitana de Guadalajara (ZMG), en comparación con estudios fitoquímicos. Se rescatan datos sobre la práctica médica herbolaria con técnicas de antropología cognitiva. Las especies más utilizadas son: árnica, cuachalalate, tila, gordolobo, salvia, cola de caballo y boldo. Los resultados demuestran que existe consenso sobre las plantas medicinales que se usan, su efectividad y la conveniencia económica en contraste con las medicinas de patente. Se enfatiza la importancia de la comprensión del uso y significado de la enfermedad, y de la adquisición del conocimiento a través de generaciones por parte de los hierberos como un patrón de conocimiento cultural.

PALABRAS CLAVE: plantas medicinales, consenso cultural, hierberos, Zona Metropolitana de Guadalajara, etnobotánica

Knowledge and Use of the Medicinal Plants in the Metropolitan Area of Guadalajara, Mexico This research emphasizes the popular use given to medicinal plants utilized by herbolarios, hierberos, yerberos or yerbateros in some markets of the Metropolitan Area of Guadalajara, as compared through phytochemical studies. Cognitive anthropology techniques were applied to compile data about medical herbal practices. The species used more often are: árnica, cuachalalate, tila, gordolobo, salvia, cola de caballo, and boldo. The results revealed the existence of a cultural consensus on the use of medicinal plants, their effectiveness and their economic benefit against the patent medicines. The importance of understanding the use and the meaning of the disease and the acquisition of knowledge among the hierberos generations is emphasized as a pattern of cultural knowledge.

KEYWORDS: medicinal plants, cultural consensus, hierberos, metropolitan area of Guadalajara, Ethnobotany

Javier E. García de Alba García: Unidad de Investigación Social, Epidemiológica y de Servicios de Salud, Instituto Mexicano del Seguro Social, Guadalajara, México javier_91046@yahoo.com

Blanca C. Ramírez Hernández: Centro Universitario de Ciencias Biológicas y Agropecuarias, Universidad de Guadalajara, Guadalajara, Jalisco, México bramirez@cucba.udg.mx

Gilberto Robles Arellano: Unidad de Medicina, Bomberos y Protección Civil de Guadalajara, Guadalajara, Jalisco, México groblesare@hotmail.com

\section{Julia ZaÑudo HernándeZ: Centro Universitario de Ciencias Biológicas y Agropecuarias, Universidad de Guadalajara, Guadalajara, Jalisco, México jzanudomx@yahoo.com.mx \\ Ana L. Salcedo Rocha: Centro Universitario de Ciencias Biológicas y Agropecuarias, Universidad de Guadalajara, Guadalajara, Jalisco, México analeticia_salcedorocha@yahoo.com.mx}

Javier E. García de Alba Verduzco: Centro Universitario de Ciencias Biológicas y Agropecuarias, Universidad de Guadalajara, Guadalajara, Jalisco, México javierdealba@gmail.com

Desacatos, núm. 39, mayo-agosto 2012, pp. 29-44

Recepción: 21 de febrero de 2011 / Aceptación: 15 de abril de 2011

\footnotetext{
* Los autores agradecen a los revisores anónimos y al editor sus valiosas sugerencias para mejorar el documento, así como a los informantes por su participación.
} 


\section{INTRODUCCIÓN}

$\mathrm{E}$ 1 uso de medicinas alternativas como las plantas medicinales y los suplementos dietarios ha sido una práctica tradicional que no ha caído en desuso (Barthelson et al., 2006: 566574 ). Se estima que $80 \%$ de la población mundial depende de remedios herbolarios tradicionales y que al menos 35000 especies vegetales presentan potencial para uso medicinal (Annan y Houghton, 2007: 141-144). La gran diversidad vegetal y la amplia riqueza cultural de México han favorecido el aprovechamiento de las plantas con fines medicinales desde épocas prehispánicas (Martínez, 1996). Este patrimonio cultural se ha transmitido de generación en generación, de manera que algunas costumbres subsisten y son ejercidas de manera cotidiana, tanto en áreas rurales como urbanas (Bye y Linares, 1987: 200-230; Campos, 1993; Yeh et al., 2003: 1277-1294). Estas prácticas médicas permanecen vigentes debido a que, entre otras cosas, los tratamientos tradicionales están basados en la enfermedad como es concebida dentro de su cultura, por lo que es pertinente percibir el tratamiento tradicional como un aspecto integrado en ella (Ryesky, 1976).

En México existe una extensa variedad de tratamientos fitoterapéuticos que forman parte de la herbolaria tradicional mexicana. Soportada por aproximadamente 4500 especies, ésta ocupa el segundo lugar a nivel mundial en el número de plantas medicinales registradas (Martínez, 1996; Barragán, 2006). Tanto en los países desarrollados como en los que están en vías de desarrollo, el uso y la comercialización de fitofármacos y productos naturales con fines medicinales muestran un crecimiento acelerado en los últimos años (Chávez y Roldán, 1994), lo que se evidencia con el aumento significativo en la demanda mundial por estos productos. También se ha comprobado que algunas plantas que se utilizan con fines medicinales tienen principios activos que se emplean para la elaboración de fármacos comerciales (Estrada, 1989: 43-46; Méndez, 2000: 5). El objetivo de este trabajo es hacer una revisión del conocimiento popular y científico del uso de las plantas medicinales consumidas con mayor frecuencia en los principales mercados de la Zona Metropolitana de Guadalajara (ZMG). La importancia de realizar este trabajo radica en el reconocimiento cultural y fitoquímico de la medicina tradicional, que aportará elementos para el aprovechamiento de este recurso. Dadas las condiciones de la cobertura de salud, la medicina tradicional es una alternativa viable que representa al mismo tiempo una herramienta para el equipo de salud y para los usuarios.

\section{METODOLOGÍA}

Este trabajo parte de una reflexión teórica-metodológica que pretende comprender procesos, más que explicar asociaciones causales. El objetivo establecido fue la base para entender el uso y el conocimiento popular de las plantas medicinales, que complementa el conocimiento producido por investigaciones fitoquímicas y/o clínicas. Se utilizaron técnicas cualitativas para rescatar los datos - opiniones, conceptos, creencias, conocimientos, actitudes- obtenidos de los informantes acerca del proceso de la práctica médica herbolaria. Se aplicó un cuestionario semiestructurado y semidirigido (Babbie, 1988) que ofreció la ventaja de que los informantes respondieron con temáticas similares, de manera que el análisis de los datos se manejó bajo una misma pauta. Las preguntas de dicho instrumento no fueron restrictivas, ya que la técnica empleada fue la entrevista semiabierta (véase Anexo 1), misma que sirvió como guía. En las preguntas se incluye una de lista libre, que consiste en solicitar la enumeración de las plantas vendidas con más frecuencia en la ZMG. Este recurso es básico para el enfoque de consenso cultural de la antropología cognitiva y con ayuda del programa Anthropac ${ }^{\oplus}$ (Borgatti, 1992) pudimos conocer la estructura del dominio semántico de ciertos conceptos, el nivel de conocimiento compartido y 


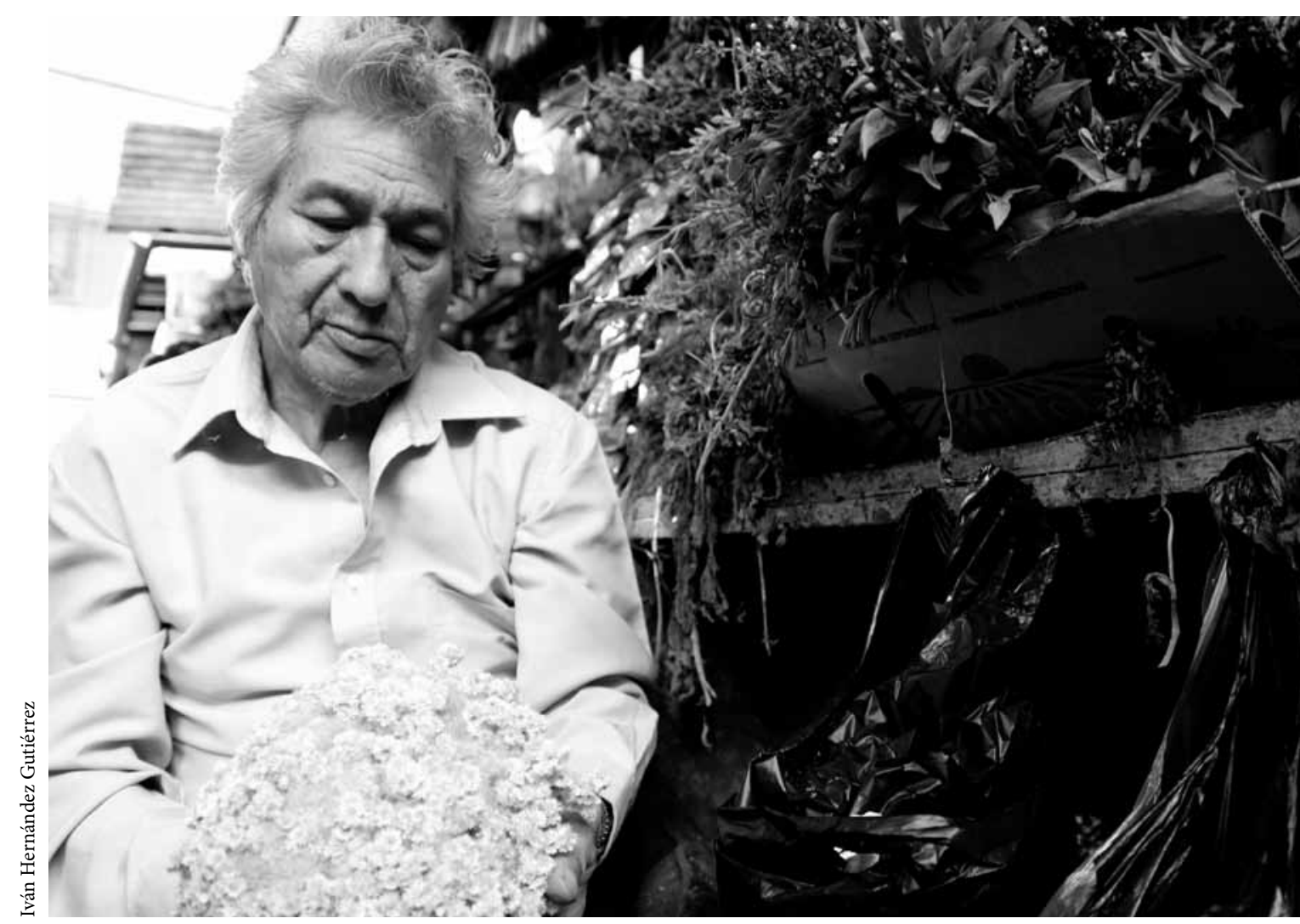

Hombre con cola de caballo, 2012.

la singularidad de este conocimiento como modelo cultural al cuantificar su variabilidad y su relevancia. El índice de relevancia de Smith, que se basa en la frecuencia de mención y el orden de aparición de cada concepto, asume que aquellos que son mencionados primero y con mayor frecuencia son los de mayor importancia para el entrevistado (Nchang, 2007: 1353). La fórmula para calcular el índice de relevancia de Smith, adoptada por Stephen P. Borgatti en el Anthropac ${ }^{\circledast}$, es:

$$
S j=l r i / n l ; S=n r j / n l
$$

Donde $r j$ es la posición de aparición del concepto $j$ dentro de la lista y $n$ es el número de veces que el concepto $j$ se encontró en la lista. Por último, $n$ es el número de conceptos que hay en la lista. El índice de relevancia general es el promedio del índice de relevancia para cada concepto según cada informante. Debido a que nos abocamos a estudiar el proceso de la práctica herbolaria tradicional, decidimos circunscribir este trabajo a los principales mercados de la ZMG registrados como uno de los puntos primordiales de distribución del recurso (Herrera, 1992), además de que representan una fuente de información y un espacio social de conocimientos y saberes tradicionales sobre plantas medicinales (Martínez Moreno et al., 2006: 79-80). Si bien existen otros espacios de distribución y venta de productos herbolarios, se procuró mantener uniformidad en el universo de trabajo, de modo que el estudio se realizó en sitios públicos y representativos de la ZMG, como el Mercado Libertad y el Mercado Corona de la 
ciudad de Guadalajara, y los mercados municipales de Tonalá, Tlaquepaque y Zapopan. Otras fuentes de obtención de plantas medicinales de uso frecuente son las tiendas de productos naturistas y farmacias de genéricos, el cultivo para uso personal o familiar y la colecta directa. Plantas que tienen uso culinario como el nopal y el joconoxtle pueden obtenerse en otros sitios de abasto.

En lo que se refiere a los informantes, nos apoyamos en los yerbateros, yerberos, herbolarios o hierberos, personas que se dedican a la colecta $y$ producción de hierbas y plantas medicinales y que comercian con ellas con un fin fitoterapéutico bajo el supuesto de que tienen una mayor competencia y dominio cultural sobre la utilidad y aplicación de las plantas medicinales (Pérez, 1982; Rodríguez et al., 1989: 59-64), además de considerar que estos actores proporcionarían información más puntual en lo que respecta a las plantas más vendidas y por tanto de mayor utilización. Los criterios de inclusión fueron: 1) expendedores - mayores de edad- de plan-San Juan de Dios-, Tlaquepaque, Tonalá y Zapopan; 2) tener un establecimiento fijo; 3 ) experiencia de más de tres años vendiendo plantas medicinales y mínimo seis meses en ese puesto - anteriores e inmediatos al estudio-, y 4) participar de forma voluntaria.

\section{RESULTADOSY DISCUSIÓN}

El estudio de las plantas medicinales no tiene como único cometido el enfoque botánico, sino que debe retomar los aspectos culturales gracias a los cuales se han acumulado estos conocimientos. Un elemento relevante en torno al uso de las plantas medicinales es la conjunción de la experiencia de la tradición y el conocimiento científico. Es importante señalar que el eje de esta investigación es la comprensión cultural de la terapéutica herbolaria, de forma tal que el enfoque fenomenológico respalde teóricamente las interpretaciones subjetivas de los herbolarios,

las cuales se emiten bajo un contexto cultural. Para demostrar la efectividad de los medicamentos vegetales recurrimos a explicaciones fitofarmacológicas, así que la relación entre propiedad medicinal y significado cultural queda evidenciada y justificada. Para este fin la metodología cualitativa, llevada a efecto con las técnicas ya descritas, se seleccionó como la más adecuada, puesto que permite indagar el significado, el conocimiento y las actitudes relacionadas con el uso de la terapéutica herbolaria, de modo que la comprensión subjetiva subyacente a esta investigación sea tratada de una forma científica, en particular en el ámbito de la salud pública. En este apartado se reportan los cuestionamientos del Anexo 1, concebidos como pertinentes para contestar las preguntas de investigación.

Se incluyeron las respuestas de ocho informantes considerados clave a partir de los resultados de las encuestas aplicadas y según los criterios de inclusión del estudio, principalmente el tiempo que se han desempeñado como expendedores de plantas medicinales que prescriben a sus clientes y cuyo modus vivendi es la herbolaria. Partimos de la idea de que estos informantes agrupan conocimientos herbolarios de toda una comunidad (Harris, 1974; Martínez et al., 2006: 79-80) y, siguiendo a Weller y Rommey (1988: 69-79), conforman una muestra suficiente para detectar un mínimo de $25 \%$ de acuerdo con $85 \%$ de validez en términos de consenso cultural.

Una proporción de $62.5 \%$ de los entrevistados fueron mujeres y $37.5 \%$, hombres. Sus edades fluctuaron entre los 22 y los 65 años, con promedio de 32 años. La escolaridad de $25 \%$ de los entrevistados fue de secundaria, $25 \%$ contaba con educación primaria y $50 \%$ carecía de estudios. De las personas entrevistadas, $75 \%$ nacieron en la ciudad de Guadalajara y $35 \%$ en poblaciones del estado de Jalisco. La mayoría de los informantes aprendió el uso de las plantas medicinales porque es un negocio familiar y sus padres o abuelos les transmitieron esos conocimientos. En general se menciona a un solo sujeto como la persona que enseña, que no sólo distribuye 
las plantas, sino también "cura o receta". Por otro lado, $62.5 \%$ de los entrevistados aseguraron que, además de las enseñanzas familiares, aprendieron de libros. Este esquema concuerda con la teoría de la fenomenología, que postula que el mundo en que nos desenvolvemos fue creado por nuestros predecesores y nos es dado para experimentarlo e interpretarlo, lo que se revela en la sucesión de los antepasados en este tipo de tradición familiar: observan, experimentan, aprenden, finalmente aprueban y continúan con la tradición.

Los entrevistados confirmaron lo reportado sobre la transmisión del conocimiento de la medicina tradicional de generación en generación (Acosta, 1993) y lo propuesto por Schutz, quien plantea que nos encontramos en un mundo previamente estructurado (Ritzer, 1993: 263-297), al exponer entre los motivos para vender plantas medicinales haber "heredado" esta tradición, la cual se practica hasta adquirir la experiencia suficiente para poder ejercerla:

Me dedico a vender plantas medicinales porque mi madre me enseñó desde pequeña y porque es muy satisfactorio poder ayudar a la gente y que las personas me busquen para que las cure de sus males. Me enseñé con mi madre, de estar observando lo que ella realizaba. Le preguntaba sobre la utilidad de cada planta y no estudié en ningún libro. Me enseñó a reconocerlas y conocer todos los nombres de cada una y utilizarlas para curar las enfermedades. Comencé a practicar en San Juan de Dios con mi madre desde los ocho años, le ayudaba a poner los nombres a las plantas y comencé a recetar a los 16 años. Tardé 12 años en aprender bien porque son cientos de plantas y cada una de ellas tiene de cinco a ocho nombres (entrevista).

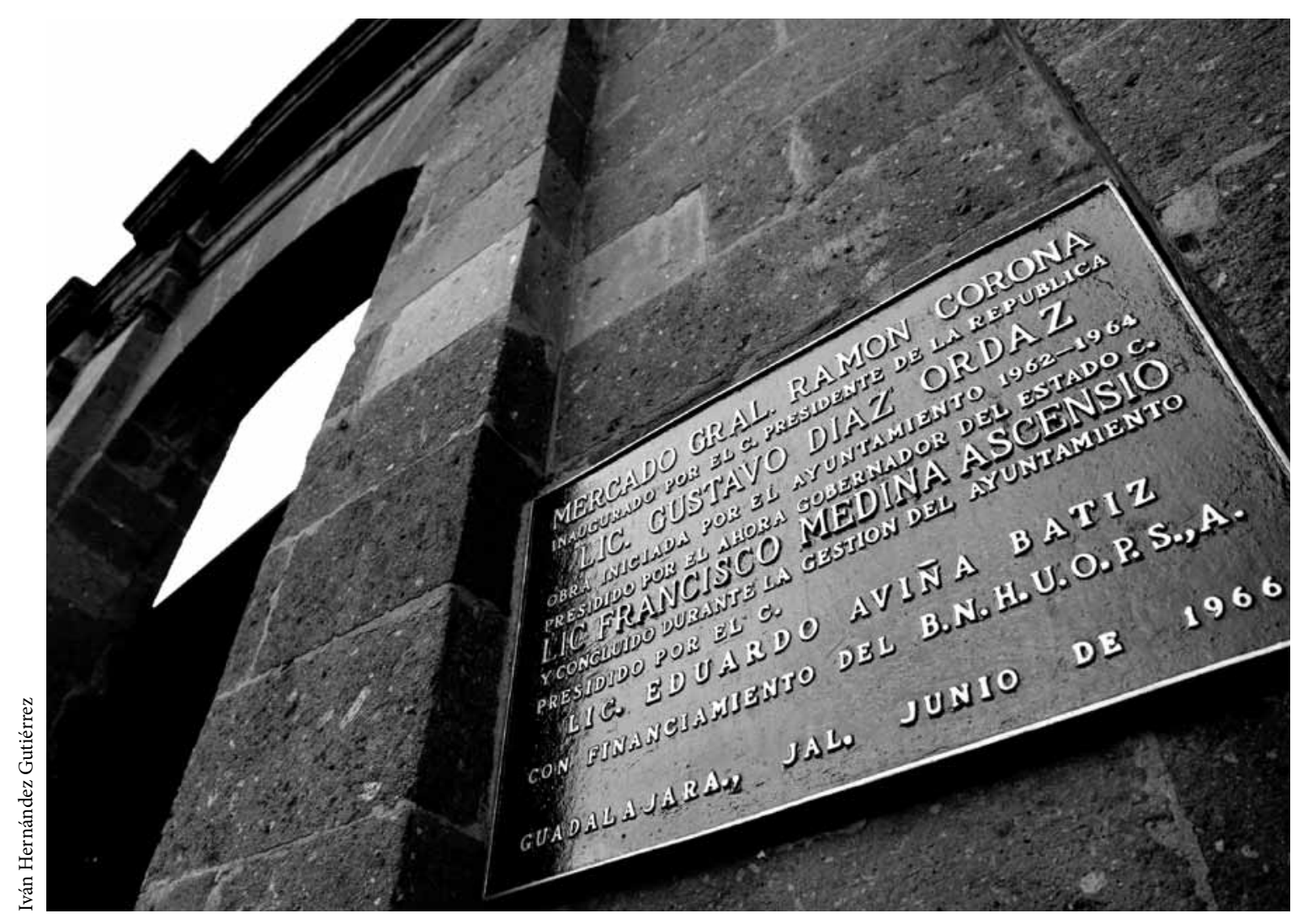

Placa del mercado, 2012. 
Ayudar a la gente lleva implícito el beneficio de poder "extraer medios en la vida o de seguir trabajando". De esta manera la herbolaria se presenta como un patrón de conducta cultural. Con base en las respuestas se infiere que la herbolaria representa un tratamiento natural y efectivo, que proporciona una manera de saber qué enfermedades se padecen y cómo curarlas, lo que indica que es una práctica terapéutica aprobada por los usuarios. $\mathrm{Al}$ mismo tiempo es un modo de mantener la identidad como pueblo, hace evidente una forma de poder $y$ autoridad que se manifiesta en el dominio de la naturaleza - conocimiento de los nombres y propiedades de las plantas para uso medicinal- y de la salud-enfermedad del individuo, sobre los demás y sobre sí mismos, como herbolario y como comprador - según la percepción de los herbolarios, 75\% de los compradores sabe qué va a adquirir-, es decir, la gente ejerce el poder del conocimiento al aplicar una terapéutica para sus problemas de salud, como una forma de autonomía sobre su propia idiosincrasia:

La mayoría de las personas llega y pregunta por una planta, y ya uno mismo les dice con qué combinar esa planta para que les sirva mejor. Es poco frecuente que vengan a preguntarme qué tomar para aliviar alguna enfermedad (entrevista).

Puede deducirse a su vez que la mayoría de las personas que acuden a estos expendios "son gente de escasos recursos": "bueno, viene de toda, pero más que nada de escasos recursos, viene como un $80 \%$ de escasos recursos y la demás es de más recursos" (entrevista). Entre las explicaciones sobre cómo y por qué curan las plantas, algunos informantes expresaron que tienen "propiedades curativas" y que son un producto natural, aunque la respuesta generalizada fue que curaban por cuestión de fe, es decir, se palpa la propuesta de que el proceso salud-enfermedad es considerado como un acontecimiento cultural, en el que la relación hombre-medicina tradicional es mediada por el conjunto de creencias, valores y signos propios de la cultura de la que forma parte (Rodríguez et al., 1989: 59-64).

\begin{abstract}
De aquí se saca la medicina, tiene sus propiedades curativas cada planta. También influye mucho la fe de la persona, en todo, hasta en el mejor doctor, si tú le pones fe, si no está tu fe de por medio lógicamente te vas con la desconfianza. Se programa, se bloquea, mentalmente se bloquea uno si no le tiene confianza a su médico. La enfermedad es una cosa que viene por la edad de uno, una descompensación del organismo que viene por su edad, la misma palabra lo dice: enferme-edad, enfermedades según la edad de la persona. Es la descompensación que tiene en el organismo, que muchas veces se descuida por el ritmo de vida que lleva uno, por la alimentación que lleva uno también, es psicológica también (entrevista).
\end{abstract}

Lo anterior nos lleva a reflexionar en torno a cómo es entendida la enfermedad en nuestro sistema hegemónico de "medicina curativa" y, por ende, respecto de la forma de recuperar la salud. El ajuste entre la medicina tradicional y la postura oficial ocurrirá en la medida en que se revalore el legado cultural de la medicina tradicional para incorporar sus saberes en la práctica médica (Bodeker, 1994: 5-16; Viesca, 1992, 1993). En este sentido es de destacar la propuesta de Lozoya (1994) de elaborar un "Cuadro básico de medicamentos" sobre plantas medicinales, el cual satisfaría la necesidad de dar a conocer las propiedades de un grupo de plantas utilizadas comúnmente que pueden catalogarse como medicamentos alternativos. Dado que la etnomedicina es parte importante de la dimensión cultural y, entre otras cosas, se tratan las creencias producto de la interacción del hombre con su ambiente - en este caso, con las plantas-, es indispensable proveer la evidencia empírica de estas creencias para que el saber popular quede confirmado como conocimiento científico basado en estudios fitoquímicos probados con estudios preclínicos y/o clínicos. En el cuadro 1 se especifica el principio activo de las plantas comercializadas con mayor frecuencia - en consenso con los entrevistados (véase cuadro 2) - en la ZMG, 
Cuadro 1. Nombre científico, empleo y principio activo/sustancias

importantes/extracto de las plantas medicinales comercializadas con más frecuencia en los mercados municipales de la Zona Metropolitana de Guadalajara

\begin{tabular}{lll}
\hline \multicolumn{1}{c}{ Nombre común $^{a}$} & \multicolumn{1}{c}{ Nombre científico $^{b}$} & \multicolumn{1}{c}{ Empleo $^{c}$} \\
\hline $\begin{array}{l}\text { Árnica (árnica } \\
\text { mexicana) }\end{array}$ & $\begin{array}{l}\text { Heterotheca inuloides } \\
\text { Cass. }\end{array}$ & Antiinflamatorio \\
\hline Cuachalalate & $\begin{array}{l}\text { Amphypterigium } \\
\text { adstringens Schl. }\end{array}$ & $\begin{array}{l}\text { Para quemar grasa, } \\
\text { colitis }\end{array}$ \\
\hline Tila & $\begin{array}{l}\text { Tilia mexicana Schl. } \\
\text { Tilia sp. }\end{array}$ & $\begin{array}{l}\text { Regula presión, para los } \\
\text { nervios }\end{array}$ \\
\hline
\end{tabular}

Principio activo/sustancias importantes/ extracto $^{d}$

Sesquiterpenos (e. g. cariolan-1, 9 $\beta$-diol,

7-Hidroxicadali, 7-hidroxi-3,

4-dihidrocaladin, cariofileno-óxido (antifúngico) (Kubo et al., 1994; Maldonado et al., 2008)

Extracto actúa como antimicrobiano (Navarro et al., 1996) y antiinflamatorio (Báez, 2007: 36-48)

Extracto efecto ansiolítico (Viola et al., 1994) beta-sitosterol (Aguirre-Hernández et al., 2007 a y b; Báez, 2007: 36-48)

$\begin{array}{lll}\text { Cola de caballo } & \begin{array}{l}\text { Equisetum hyemale L. } \\ \text { E. robustum Schletcht \& } \\ \text { Cham. }\end{array} & \begin{array}{l}\text { Enfermedades renales } \\ \text { (limpia los riñones) }\end{array} \\ \end{array}$

Extracto (Park y Jeon, 2008) efecto hipoglucemiante de derivados de canferol glucosilado y glucósido cafeil (Negri, 2005)

Flavonoides (Planta Medica, 1994;

$\begin{array}{ll}\text { Gordolobo } & \text { Gnaphalium sp. } \\ \text { G. conoideum Kunth. Tos, asma, bronquitis }\end{array}$
Campos-Bedolla et al., 2005) propiedades relajantes (Rojas, Tortoriello y Navarro, 2001; Rodríguez-Ramos y Navarrete, 2009)

\begin{tabular}{lll}
\hline \multirow{2}{*}{ Salvia } & $\begin{array}{l}\text { Salvia officinalis } L . \\
\text { Salvia sp. } \\
\text { Lippia geminata H.B.K. }\end{array}$ & \\
&
\end{tabular}

Linuron (Vaverkova, Holla y Teckel, 1995); componentes gastroprotectores (Kamatou et al., 2008; Mayer et al., 2009)

\section{Siete azahares (magnolia, zapote blanco, azahar, toronjil, tres tilas) \\ Magnolia officinalis \\ Cedronella mexicana \\ Benth. \\ Citrus sp. C. medica L. \\ Agastache mexicana \\ Tilia sp.}

\begin{tabular}{lll}
\hline Damiana & Turnera diffusa Willd. & $\begin{array}{l}\text { Sistema nervioso, } \\
\text { afrodisiaco }\end{array}$ \\
\hline Boldo & Peumus boldus Molina & $\begin{array}{l}\text { Enfermedades hepáticas } \\
\text { y renales }\end{array}$
\end{tabular}

Para los nervios

Honokiol (acción sobre sistema nervioso)

(Planta Medica, 1995). Limoneno, vitamina $C$, hesperidina, tiramina, N-metiltiramina (Ortiz y Browner, 1985; Aguirre-Hernández et al., 2007 a y b; Young-Jung et al., 2011; Hyun y Song, 2011). Antihipertensivo (HernándezAbreu et al., 2009)

5-hidroxi-7, 3',4'-trimetoxi-flavona (Dominguez e Hinojosa, 1976; AlcarazMeléndez, Real-Cosío y Robert, 2002: 293-301)

Boldine (antioxidante) (alcaloide) (Speisky y Cassels, 1994; O’Brien, Carrasco-Pozo y Speisky, 2006)

Arbutina, ácido cítrico (Martínez y Martínez, 1992); nutrición y metabolismo (Manzanero-Medina et al., 2009)

\footnotetext{
${ }^{\text {a }}$ Obtenido de los informantes.

b Obtenido de revisión bibliográfica.

${ }^{\mathrm{c}}$ Obtenido de los informantes, pero interpretado por los autores de este trabajo.

${ }^{\mathrm{d}}$ Obtenido de revisión bibliográfica.
} 
así como su uso según la tradición mexicana. Esto podría coadyuvar a la solución de un problema de salud pública bien conocido: la cobertura de los servicios de salud y el abasto insuficiente de medicamentos. La propuesta resulta una opción real para un país en vías de desarrollo con una gran tradición herbolaria.

Las plantas medicinales mencionadas con mayor frecuencia (véase cuadro 2), y por tanto las enfermedades que más se tratan, tienen asociación con los principales motivos de atención (véase cuadro 3 ) reportados para Jalisco en municipios con alta densidad poblacional (ssJ, 2009). Sin embargo, hay que aclarar que existen otras plantas que los usuarios cultivan o que se obtienen en otros expendios, como la zanahoria y la sábila que se emplean para curar la gastritis y no se compran generalmente en una hierbería. También hay otras terapéuticas que pueden aplicarse de manera conjunta.

En ocasiones, cuando se preguntaba a los entrevistados el modo de empleo de las plantas, había re- todo si se trataba de algún compuesto de varias plantas, puesto que se considera un secreto de trabajo. Asimismo los expendedores de plantas medicinales consideran que la gente acude a este tipo de terapéutica, entre otras razones, porque resulta más barata comparada con la medicina de patente. $\mathrm{Al}$ cuestionar a los informantes por qué las plantas medicinales curan, contestaron según la propuesta de Ryesky (1976), que afirma que este tipo de terapia está vigente porque el proceso de enfermedad y curación es congruente con su esquema cultural. Esto apoya a su vez lo expuesto por la fenomenología en cuanto a que las prácticas médicas curativas son válidas porque rinden resultados provechosos, debido en gran parte a que los pacientes se recuperan porque las enfermedades siguen su curso y porque los tratamientos tradicionales están basados en la enfermedad como es concebida en su cultura, por lo que es pertinente percibir el tratamiento tradicional como un aspecto integrado a ella.

Es importante destacar la experiencia de atención primaria en salud en otros países. En China el uso

Cuadro 2. Estructura del modelo semántico de principales plantas medicinales vendidas en los mercados municipales de Guadalajara, Tlaquepaque, Tonalá y Zapopan

\begin{tabular}{|c|c|c|c|}
\hline Planta & Porcentaje de mención & Indice de relevancia & Lugar \\
\hline Árnica & 100 & 0.642 & 1 \\
\hline Cuachalalate & 75 & 0.514 & 2 \\
\hline Tila & 63 & 0.406 & 3 \\
\hline Gordolobo & 50 & 0.313 & 4 \\
\hline Salvia & 50 & 0.267 & 5 \\
\hline Cola de caballo & 50 & 0.265 & 6 \\
\hline Pingüica & 38 & 0.191 & 7 \\
\hline Boldo & 38 & 0.187 & 8 \\
\hline Siete azahares & 50 & 0.178 & 9 \\
\hline Damiana & 38 & 0.065 & 10 \\
\hline \multicolumn{2}{|c|}{ Conocimiento compartido promedio } & 0.55 & \\
\hline \multicolumn{2}{|c|}{ Razón de variabilidad } & $>3$ & \\
\hline
\end{tabular}

Fuente: Lista libre. 
Cuadro 3. Principales motivos de atención y posible relación con plantas medicinales, Jalisco, 2009

\begin{tabular}{|c|c|c|c|}
\hline Lugar & Motivo de atención ${ }^{a}$ & $\begin{array}{l}\text { Tasa por } \\
100000 \\
\text { habitantes }\end{array}$ & $\begin{array}{l}\text { Plantas medicinales asociadas }{ }^{b} \text { a las } \\
\text { atenciones reportadas por la ssJ }\end{array}$ \\
\hline 1 & Infecciones respiratorias agudas altas & 17930.1 & $\begin{array}{l}\text { Gordolobo, eucalipto, rosa de Castilla, } \\
\text { damiana }\end{array}$ \\
\hline 2 & Enfermedades infecciosas intestinales & 4829.6 & $\begin{array}{l}\text { Cuachalalate, salvia, quina roja, rosa } \\
\text { de Castilla, olivo }\end{array}$ \\
\hline 3 & Infección de vías urinarias & 2747.1 & $\begin{array}{l}\text { Cola de caballo, pingüica, palo de Brasil, } \\
\text { jojoba, palo dulce, prodigiosa }\end{array}$ \\
\hline 4 & Úlceras, gastritis y duodenitis & 1029.2 & Sangre de drago, manzanilla, linaza \\
\hline 5 & Hipertensión arterial & 664.8 & $\begin{array}{l}\text { Tila, siete azahares, zapote blanco, melisa, } \\
\text { indirectamente: tranchalagua, cocolmeca, } \\
\text { raíz de lima }\end{array}$ \\
\hline 6 & Diabetes mellitus & 534.0 & Nopal, chaya, matarique \\
\hline 7 & Otitis media aguda & 532.0 & Árnica, eucalipto \\
\hline 8 & Asma y estado asmático & 371.8 & Gordolobo, eucalipto, tila, salvia \\
\hline 9 & $\begin{array}{l}\text { Accidentes (de tráfico de vehículo } \\
\text { de motor) [traumatismos] }\end{array}$ & 313.4 & Árnica \\
\hline
\end{tabular}

a Fuente: Secretaría de Salud de Jalisco.

${ }^{\mathrm{b}}$ Fuente: Lista libre.

de plantas medicinales tiene una influencia determinante. Su política de industrialización de medicamentos ha permitido atender los requerimientos básicos de una población de casi 1400 millones de habitantes. El modelo chino combina el uso de la "medicina tradicional" y de la "occidental", y los resultados son altamente eficaces —en 1949 la tasa de mortalidad era de 25 por 1000 habitantes, disminuyó a 6.2 por 1000 habitantes en 1978 y en 2004 era de 7.8 por 1000 habitantes-, por lo que la Organización Mundial de la Salud (OMs) utilizó ideas de este modelo en su campaña para alcanzar las metas del plan "Salud para Todos en el Año 2000" y dio nacimiento en México al programa "Promoción y Desarrollo de la Medicina Tradicional" (Lozoya, 1994: 365-371). En nuestro país, algunas universidades y el Instituto Mexicano del Seguro Social (IMSs) realizan labores de investigación en el área de las plantas medicinales e incluyen ciertos medicamentos vegetales, aunque se requiere un esfuerzo mayor para complementar con terapéuticas consensadas por la propia población, de manera que el paciente y el médico tengan más de una alternativa para procurar la salud (oms, 2002).

\section{REFLEXIONES FINALES}

La herbolaria es un recurso básico de la medicina popular, pero nuestro conocimiento de ella es escaso e impreciso. Estamos lejos de asegurar un rescate apropiado de este flanco de la cultura. El "retorno" a la naturaleza que anuncia la sociedad de consumo con una gran variedad de productos de origen vegetal puede conducirnos no necesariamente a conservar la tradición o aplicación de tratamientos de manera tradicional, sino a buscar extractos o principios activos para la elaboración de suplementos o de medicinas alopáticas de patente, lo que implica nichos de oportunidad y de peligro para esta práctica 
medicinal. La nomenclatura de las plantas que se utilizan actualmente en la medicina tradicional mexicana es un sincretismo entre varias culturas, por ejemplo: la cultura médica indígena -estafiate, zapote blanco, epazote, zoapatle, toloache, etc.-, la cultura europea - ajenjo, árnica, toronjil, manzanilla, hierbabuena, tomillo, romero, etc.-, la árabe —albahaca, alucena, aloe, etc.—, la asiática — té verde, canela, etc.- (Lozoya, 1987: 83-94).

A lo largo de la historia, la población mexicana, sobre todo la indígena, seguida de la mestiza, sobrevivió recurriendo a la medicina herbolaria, cuyos recursos pasaron a formar parte de la medicina popular y doméstica, dado que solucionaban muchos de los problemas de salud de su tiempo y espacio, únicamente condicionado su uso por la cultura y la ecología, por lo que llegó a tener un estatus nacional. Sin embargo, la aplicabilidad a la medicina de los recursos de la herbolaria sigue siendo inagotable. Por ejemplo, se ha impulsado el uso terapéutico de la Opuntia sp. para el tratamiento de la diabetes mellitus (Frati et al., 1987; Andrade-Cetto y Wiedenfeld, 2010: 940-943). En la actualidad la medicina doméstica se ha limitado por el proceso de urbanización e industrialización. El conocimiento terapéutico de las plantas se ha relegado - sobre todo de aquellas cuyo manejo requiere experiencia diagnóstica y terapéutica- a un estrato de actores especializados, como los hierberos, los curanderos, las parteras, los sobadores, que evidencian un profundo dominio de la medicina tradicional, aún no comprendido cabalmente por la medicina hegemónica (Lozoya, 1987: 83-94).

Otros aspectos que no se contemplan en las estadísticas oficiales son el dolor, el sufrimiento y la desesperación causados por las afecciones agudas $\mathrm{y}$ crónicas que no son tratadas o no pueden recibir tratamiento de acuerdo con la cultura y que provocan una disminución de los años de vida y de la calidad de la misma en los grupos sociales que acceden a medicinas marginales (Browner, Ortiz de Montellanos y Rubel, 1992: 223-263). Estos problemas han sido señalados y debatidos desde hace tiempo por investigadores como Gamio (1966), Mendizábal (1947), Cravioto (1979: 244-248), López Cámara, (1968: 355-367), Collado (1983: 243-262), Lozoya y Zolla (1983). Dichas soluciones a favor de la medicina llamada tradicional enfrentan a una medicina hegemónica que invade campos y no comparte espacios de atención - hay pocos ejemplos que confirman la excepción-, y menos el marco conceptual que los soporta. Un ejemplo es el servicio social en ciencias de la salud, proceso durante el cual los pasantes reciben poco entrenamiento en aspectos sociales, económicos y culturales de las prácticas médicas tradicionales de las comunidades a las que son asignados (Aguirre, 1986: 234), además de que la ausencia de un lenguaje común entre los médicos científicos y los tradicionales es un obstáculo formidable.

$\mathrm{Al}$ interior del paradigma científico, el conocimiento se acumula a través de métodos y procedimientos estandarizados, precisos y replicables, cuyo resultado es un cuerpo de conocimientos de base empírica sobre la estructura y función del objeto de estudio. Examinar los componentes de los sistemas etnomédicos a la luz de las categorías científicas permite entenderlos de manera parcial y a la inversa. En otras palabras, estamos ante un caso de inconmensurabilidad, como señala Khun (1975). Al respecto Browner, Ortiz de Montellanos y Rubel (1992: 223263) proponen:

1) Detectar los fenómenos registrados con categorías emic. Por ejemplo, "al determinar las características de un síndrome nosológico de tipo tradicional y enlistar las razones por las cuales se usa un tipo de plantas medicinales al surgir síntomas o enfermedades específicas.

2) Determinar hasta qué punto pueden entenderse los fenómenos descritos en términos de concepciones y metodologías científicas. Por ejemplo: con base en la afirmación de los representantes de medicina tradicional, que una planta es efectiva para determinada enfermedad o provoca 
ciertos efectos específicos. Una evaluación de tipo etic compartiría el análisis farmacológico correspondiente para determinar si sus compuestos se relacionan con los efectos señalados, $v$. gr.: remedios para que detengan el sangrado postparto contra la atonía uterina.

3) Detectar áreas de convergencia o divergencia entre los fenómenos estudiados y los esquemas interpretativos biológico-científicos, $v$. gr.: zapote blanco e hipotensión.

En el caso de las plantas medicinales la mayor parte de la literatura atribuye los efectos curativos a su valor simbólico, al carisma del terapeuta o a un efecto placebo. Incluso pueden detectarse efectos nocivos si se subordina el poder curativo al ámbito biológico, lo que produce un conocimiento rudimentario sobre la verdadera eficacia de las plantas medicinales, puesto que apenas se inician procedimientos sistemáticos integrales para evaluarlas. Ortiz de Montellanos y Browner propusieron (1985: 57-88) un procedimiento básico: 1) identificación botánica precisa y completa; 2) investigación bibliográfica ex- haustiva sobre la bioquímica de los principios activos de la planta; 3) documentación farmacológica de los efectos fisiológicos de la planta, y 4) comparación de los efectos terapéuticos conocidos por la ciencia con aquellos que los usuarios de las plantas desean lograr y así evaluar congruencias y discrepancias. Obviamente, estos aspectos no agotan las evaluaciones posibles para las plantas medicinales, máxime si se toma en cuenta que los avances tecnológicos son cada vez más rápidos. El esquema anterior tiene la ventaja de que es gradual y acumula conocimiento, y establece a su vez un puente entre la medicina tradicional y la medicina científica, además de que puede aplicarse por regiones fitotípicas, a manera de protocolo-inventario a cargo de las universidades públicas de cada estado a partir de estudios comunitarios del uso de plantas medicinales, colectas dirigidas y estudios laboratoriales. En el cuadro 4 se presenta un ejemplo breve de la aplicación del esquema en plantas utilizadas durante el parto, primer motivo de atención hospitalaria del sector público (Valadez, Alfaro y Pozos, 2003).

Cuadro 4. Resumen evaluatorio de plantas útero-activas

\begin{tabular}{|c|c|c|c|c|c|c|}
\hline $\begin{array}{c}\text { Explicación } \\
\text { emic }\end{array}$ & $\begin{array}{l}\text { Planta } \\
\text { referida }\end{array}$ & $\begin{array}{l}\text { Nombre } \\
\text { botánico }\end{array}$ & $\begin{array}{l}\text { Irritante } \\
\text { medicina } \\
\text { tradicional }\end{array}$ & $\begin{array}{c}\text { Sustancias } \\
\text { químicas } \\
\text { irritantes }\end{array}$ & $\begin{array}{c}\text { Sustancias } \\
\text { activas }\end{array}$ & $\begin{array}{c}\text { Efectividad } \\
I=\text { baja } \\
I V=\text { alta }\end{array}$ \\
\hline \multirow{4}{*}{$\begin{array}{l}\text { Por frío-calor } \\
\text { circula la } \\
\text { sangre y la } \\
\text { irritación } \\
\text { favorece } \\
\text { contracciones }\end{array}$} & Zoapatle & $\begin{array}{l}\text { Montanoa } \\
\text { tormentosa }\end{array}$ & Sí & $(?)$ & $\begin{array}{l}\text { Zoapatanol } \\
\text { Montanol }\end{array}$ & IV \\
\hline & Ajo & $\begin{array}{l}\text { Allium } \\
\text { sativum }\end{array}$ & Sí & Aceites & Prostaglandina & III \\
\hline & Canela & $\begin{array}{l}\text { Cinnamomum } \\
\text { zeylanicum }\end{array}$ & Sí & $\begin{array}{l}\text { Aceites } \\
\text { Tanino }\end{array}$ & $\begin{array}{l}\text { Eugenol } \\
\text { Miristicina } \\
\text { Alcanfor }\end{array}$ & II \\
\hline & Cilantro & $\begin{array}{l}\text { Coriandrum } \\
\text { sativum }\end{array}$ & Sí & $\begin{array}{l}\text { Aceites } \\
\text { Tanino }\end{array}$ & $\begin{array}{l}\text { Borneol } \\
\text { Terpeno }\end{array}$ & I \\
\hline
\end{tabular}

Fuente: Browner, Ortiz de Montellanos y Rubel (1992: 223-263). 
Como se observa, esta metodología puede ayudar a realizar investigación sobre problemas prioritarios de salud a partir de una visión emic de problemas, de manera que podamos obtener resultados reproducibles. Se reconoce que la propuesta tiene limitaciones, pero también aciertos: 1) se expone la teoría emic sobre la cual se basan las propiedades de las plantas medicinales; 2) hay posibilidades de comprobar principios y resultados, y 3) manifiesta un nivel de confiabilidad alto al establecer un puente de saberes. El relativismo cultural que priva en la antropología social ha enriquecido la etnología de los materiales etnobotánicos, pero indirectamente ha empobrecido los esfuerzos por definir esquemas comparativos que permitan validar proposiciones prácticas para descubrir relaciones constantes entre variables biológicas, ecológicas, culturales y sociales, ha establecido paradigmas híbridos en los que convergen las disciplinas involucradas y ha eliminado el carácter inmutable de entidades nosológicas y de ciertos procesos. Al mismo tiempo, podríamos aclarar el papel que juega la cultura en el modo y circunstancias en que ocurre el proceso de salud-enfermedad-atención en la medicina tradicional. Además de aportar elementos para la identidad de los grupos sociales usuarios de estas plantas medicinales, podrían integrarse farmacopeas regionales, lo que aumentaría la posibilidad de que no sean desplazadas o injustamente absorbidas por las corporaciones farmacéuticas internacionales que producen fármacos más costosos y menos accesibles para la población usuaria de la medicina tradicional (Browner, Ortiz de Montellanos y Rubel, 1992: 223-263).

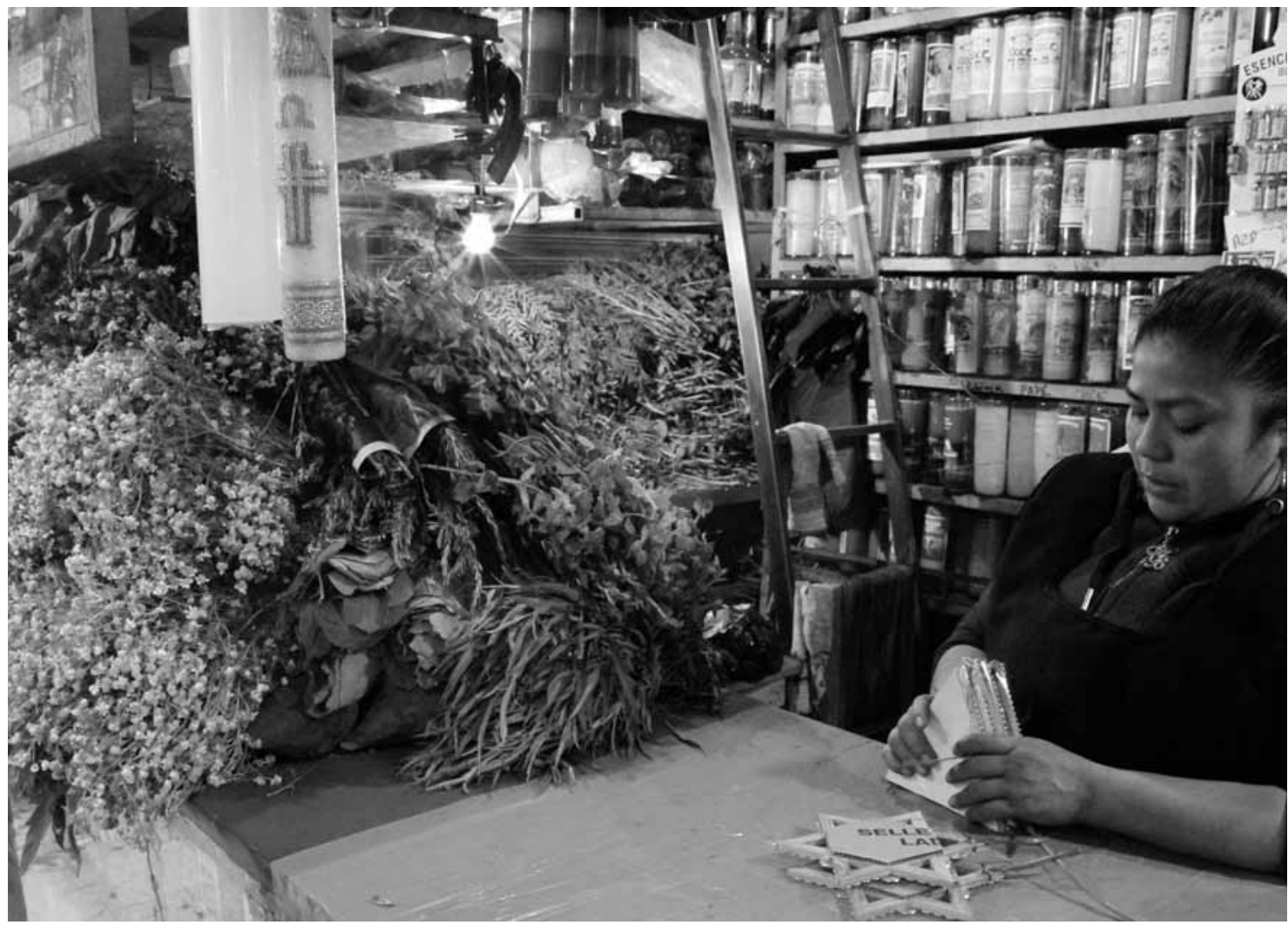

Vendedora de plantas, 2012. 


\section{Anexo 1}

\begin{tabular}{l|l}
\hline Fecha & Entrevista realizada por: \\
\hline Registro núm. & \\
\hline Mercado: & Puesto: \\
\hline
\end{tabular}

1. Nombre

2. Edad

3. Lugar de nacimiento

4. Tiempo viviendo en la ciudad de Guadalajara (si es originario de Guadalajara preguntar si ha residido en otras partes, si es así preguntar desde cuándo vive nuevamente aquí)

5. Tiempo vendiendo plantas medicinales

6. Tiempo vendiendo plantas medicinales en esa plaza

7. ¿Por qué se dedica a vender plantas medicinales?

8. ¿Qué significado tiene la herbolaria para usted?

9. ¿Cómo se enseñó a usar las plantas medicinales? (si responde que en libros preguntar de qué tipo y cuáles específicamente)

10. ¿Estudió alguna otra cosa? (preguntar escolaridad)

11. ¿Quién le enseñó cómo usarlas y cómo reconocerlas?

12. ¿Cómo practicó, en dónde y en quiénes?

13. ¿Cuánto tiempo le llevó aprender? (preguntar por qué le llevó ese tiempo)

14. ¿En qué lugar se enseñó a usarlas? (localidad y espacio físico)

15. ¿Qué tipo de gente viene a comprarle plantas medicinales? (preguntar porcentajes)

16. ¿Cuando la gente viene a comprarle plantas le pregunta cuál llevar o llega y le pide una o algunas en particular? ¿En qué porcentaje?

17. ¿Cuáles plantas, específicamente, vende con más frecuencia? (al menos 10 plantas en particular)

18. a) ¿Para qué enfermedades se emplea cada una?

En caso de que varias plantas se utilicen para una enfermedad preguntar cuál es la mejor para la enfermedad citada.

b) ¿Cómo se utilizan (cada una)? Opciones:

- ¿La planta se usa fresca o seca? ¿Cómo se almacena?

- Parte de la planta empleada

- Forma de preparación

- Frecuencia de uso

- Indicaciones y contraindicaciones

- Efectos colaterales

19. ¿Por qué cree usted que la gente compra plantas medicinales?

20. ¿Usted considera que las plantas medicinales curan igual que las medicinas de patente? ¿Por qué?

21. ¿La gente utiliza las plantas medicinales con medicamentos de patente?

a) ¿Por qué o para qué?

b) ¿Usted lo recomienda a sus clientes?

22. ¿ Por qué las plantas medicinales curan?

23. ¿Cómo curan las plantas medicinales?

24. ¿Qué significan para usted las enfermedades?

25. ¿Qué significa para usted curar con plantas medicinales? (se puede complementar la pregunta cuestionando el significado de ayudar a la gente)

26. a) ¿Cuál es el costo promedio de las plantas que le compran?

b) ¿Cuál es el mínimo y cuál el máximo?

c) ¿A la gente le parece caro o barato? 


\section{Bibliografía}

Acosta de la Luz, Lérida L., 1993, Proporciónese salud: cultive plantas medicinales, Editorial Científico Técnica, La Habana.

Aguirre Beltrán, Gonzalo, 1986, Antropología médica, Centro de Investigaciones y Estudios Superiores en Antropología Social, México.

Aguirre-Hernández, Eva et al., 2007a, "Pharmacological Evaluation of the Anxiolytic and Sedative Effects of Tilia americana L. var. mexicana in Mice", en Journal of Ethnopharmacology, vol. 109, núm. 1, pp. 140-145.

- et al., 2007b, "Bioactivity-guided Isolation of Beta-sitosterol and Some Fatty Acids as Active Compounds in the Anxiolytic and Sedative Effects of Tilia americana var. mexicana", en Planta Medica, vol. 73, núm. 11, pp. 1148-1155.

Alcaraz-Meléndez, Lilia, Sergio Real-Cosío y Manuel Robert, 2002, "Morphological Comparison of Damiana (Turnera diffusa, Willd.) Regenerated in Vitro from Leaves Cultured in Solidified Medium and Liquid Cultures", en Scientia Horticulturae, vol. 96, núm. 1-4, pp. 293-301.

Andrade-Cetto, Adolfo y Hemult Wiedenfeld, 2010, “AnLem", en Journal of Ethnopharmacology, vol. 133, núm. 2, pp. 940-943.

Annan, Kofi y Peter J. Houghton, 2007, “Antibacterial, Antioxidant and Fibroblast Growth Stimulation of Aqueous Extracts of Ficus asperifolia Miq. and Gossypium arboreum L., Wound-healing Plants of Ghana", en Journal of Ethnopharmacology, vol. 119, pp. 141-144.

Babbie, Earl R., 1988, Métodos de investigación por encuesta, Biblioteca de la Salud, Fondo de Cultura Económica, México.

Báez Castillo, Glenda, 2007, “Determinación del efecto antiinflamatorio de los extractos hexánicos, etanólicos y clorofórmicos de las plantas medicinales: Bursera aloexylon, Amphypteryngium adstringens, Tilia mexicana, Verbascum thapsus, Rosmarinus officinalis, Salvia hispanica, Aloe vera, Opuntia ficus-indica en un modelo animal", tesis de maestría en biomedicina clínica, Departamento de Ciencias Químico-Biológicas, Escuela de Ingeniería y Ciencias, Universidad de las Américas, Puebla.

Barragán Solís, Anabella, 2006, "La práctica de la autoatención por fitoterapia en un grupo de familias mexicanas", en Archivos en Medicina Familiar, vol. 8, pp. 155-162.

Barthelson, Roger A. et al., 2006, "Development of a Comprehensive Detection Method for Medicinal and Toxic Plant Species", en American Journal of Botany, vol. 4, pp. 566-574.

Bodeker, Gerard, 1994, "Savoir Tradicional et Politique de Santé Publique", en Nature et Ressources, vol. II, núm. 30, pp. 5-16.

Borgatti, Stephen Peter, 1992, Anthropac 4.0 Reference Manual, Analytic Technologies, Columbia University, Columbia.

Browner, Carole H., Bernard Ortiz de Montellanos y Arthur J. Rubel, 1992, "El análisis comparativo de sistemas médicos", en Paola Sesia (ed.), Medicina tradicional, herbolaria y salud comunitaria en Oaxaca, Gobierno del Estado de Oaxaca, Centro de Investigaciones y Estudios Superiores en Antropología Social, Oaxaca, pp. 223-263.

Bye, Robert y Edelmira Linares, 1987, "Usos pasados y presentes de algunas plantas medicinales encontradas en los mercados mexicanos", en América Indígena, vol. 47, núm. 2, pp. 200-230.

Campos Navarro, Roberto, 1993, "Estudios urbanos en México sobre el uso de las plantas medicinales", en Jesús Kumate (comp.), La investigación científica de la herbolaria medicinal mexicana, Secretaría de Salud, México.

Campos-Bedolla, Patricia et al., 2005, "Effect of Gnaphalium conoideum HBK on Guinea Pig Airway Smooth Muscle: Role of L-type Ca2+ Channels", en Journal of Ethnopharmacology, vol. 97, núm. 2, pp. 267-272.

Chávez Quiñones, Evelia y José Roldán, 1994, "Vivero de plantas medicinales”, ponencia, III Reunión Nacional de Investigación Médica, Instituto Mexicano del Seguro Social, Oaxtepec, Morelos.

Collado Ardon, Rolando, 1983, "Perfil y arraigo del médico en el medio rural en México", en Educación Médica y Salud, vol. 17, núm. XX, pp. 243-262.

Cravioto Meneses, Adalberto, 1979, "Recent Progress in the Program for Extending the Service Coverage in $\mathrm{Ru}$ ral México", en Bulletin of the Pan American Health Organization, vol. 13, núm. XX, pp. 244-248.

De la Fuente, Julio, 1941, "Creencias indígenas sobre la onchocercosis, paludismo y otras enfermedades", en América Indígena, vol. 1, pp. 43-46.

Domínguez, Xorge Alejandro y Marianela Hinojosa, 1976, "Mexican Medicinal Plants. XXVIII. Isolation of 5-hidrixi-7, 3', 4'-trimetoxi-flavona from Turnera Diffusa”, en Planta Medica, vol. 30, núm. 1, pp. 68-71.

Estrada Lugo, Erik, 1989, "Perspectiva interdisciplinaria en la investigación etnobotánica a partir del servicio: plantas medicinales", en Memorias del Tercer Coloquio 
de Medicina Tradicional Mexicana "Un Saber en Discusión”, Escuela Nacional de Estudios Superiores Zaragoza, Universidad Nacional Autónoma de México, pp. 211-228.

Frati Munari, Alberto et al., 1987, "Estudios sobre el mecanismo de acción hipoglucemiante del nopal (Opuntia sp.)", en Revista Médica del Instituto Mexicano del Seguro Social, vol. 18, pp. 7-12.

Gamio, Manuel, 1966, Consideraciones sobre el problema indígena, Instituto Indigenista Interamericano (Serie Antropología Social), México.

Harris, Marvin, 1974, Cows, Pigs, Wars, and Witches: The Riddles of Culture, Random House, Nueva York.

Hernández-Abreu, Oswaldo et al., 2009, "Antihypertensive and Vasorelaxant Effects of Tilianin Isolated from Agastache Mexicana are Mediated by NO/cGMP Pathway and Potassium Channel Opening", en Biochemical Pharmacology, vol. 78, núm. 1, pp. 54-61.

Herrera Saldaña, Bertha, 1992, “Comercialización de plantas medicinales en la ciudad de Guadalajara”, tesis de licenciatura, Facultad de Ciencias Biológicas, Universidad de Guadalajara, Guadalajara.

Hyun Kim y Mi-Jang Song, 2011, "Analysis and Recordings of Orally Transmitted Knowledge about Medicinal Plants in the Southern Mountainous Region of Korea", en Journal of Ethnopharmacology, vol. 134, pp. 676-696.

Kamatou, Guy et al., 2008, "South African Salvia Species: A Review of Biological Activities and Phytochemistry Review Article", en Journal of Ethnopharmacology, vol. 119, núm. 3, pp. 664-672.

Kubo, Isao et al., 1994, "Antimicrobial Agents from Heteroteca Inuloides", en Planta Medica, Journal of Medicinal Plant Research, vol. 60, núm. 3, pp. 218-221.

Kuhn, Thomas, 1975, La estructura de las revoluciones científicas, Fondo de Cultura Económica, México.

López Cámara, Víctor, 1968, “Organización del servicio social de los pasantes de odontología en México”, en Salud Pública de México, vol. 10, núm. XX, pp. 355-367.

Lozoya, Xavier, 1987, Medicina tradicional y atención primaria, Centro de Investigaciones y Estudios Superiores en Antropología Social México, Cuadernos de la Casa Chata, núm. 159, pp. 83-94.

1994, "La producción de medicamentos y la vigencia de la herbolaria medicinal", en Revista Médica del Instituto Mexicano del Seguro Social, vol. 32, pp. 365-371.

— y Carlos Zolla, 1983, La medicina invisible, Folios Ediciones, México.

Maldonado-López Yurishi et al., 2008, "Mexican Arnica Anti-Inflammatory Action: Plant Age is Correlated wi- th the Concentration of Anti-Inflammatory Sesquiterpenes in the Medicinal Plant Heterotheca inuloides Cass. (Asteraceae)", en Economic Botany, vol. 62, núm. 2, pp. 161-170.

Manzanero-Medina, Gladys et al., 2009, "Etnobotánica de siete raíces medicinales en el Mercado de Sonora de la ciudad de México", en Polibotánica, vol. 27, pp. 191-228.

Martínez, Maximino, 1996, Las plantas medicinales de México, Editorial Botas, México.

Martínez Bravo, Eugenio y Dora Martínez Olivares, 1992, Microdosis, una alternativa medicinal, Universidad Autónoma de Zacatecas, Zacatecas.

Martínez Moreno, David et al., 2006, "Plantas medicinales de cuatro mercados de Puebla”, en Boletín de la Sociedad Botánica de México, vol. 79, pp. 79-87.

Mayer, Barbara et al., 2009, "Gastroprotective Constituents of Salvia officinalis L., en Fitoterapia, vol. 80, núm. 7, pp. 421-426.

Menéndez Castillo, Rosa, 2000, "Acerca de las pautas de la oMs en la investigación para la evaluación de la seguridad y eficacia de los medicamentos herbarios", en Revista Cubana de Plantas Medicinales, vol. 5, núm. 1.

Mendizábal, Miguel Othón de, 1947, "Situación actual de la asistencia médica y social en la República”, en Miguel Othón de Mendizábal, Obras Completas, vol. 6, Talleres Gráficos de la Nación, México.

Navarro, Víctor et al., 1996, "Antimicrobial Evaluation of Some Plants Used in Mexican Traditional Medicine for the Treatment of Infectious Diseases", en Journal of Ethnopharmacology, vol. 53, núm. 3, pp. 143-147.

Nchang, Regina, 2007, “The Forgotten Link: Male and Female Farmers' Decision Making Frameworks in Plant Genetic Diversity Management", en African Crop Science Society, vol. 8, Camerún, pp. 1351-1362.

Negri, Giuseppina, 2005, "Diabetes Melito: Plantas e Princípios Ativos Naturais Hipoglicemiantes", en Revista Brasileira de Ciências Farmacêuticas, vol. 41, núm. 2, pp. 121-142.

O’Brien, Peter, Catalina Carrasco-Pozo y Hernán Speisky, 2006, "Boldine and its Antioxidant or Health-Promoting Properties", en Chemico-Biological Interactions, vol. 159, núm. 1, pp. 1-17.

Organización Mundial de la Salud (OMs), 2002, Pautas generales para las metodologías de investigación y evaluación de la medicina tradicional, Organización Mundial de la Salud, Ginebra.

Ortiz de Montellanos, Bernard y Carol H. Browner, 1985, "Chemical Bases for Medicinal Plant Use in Oaxaca", en Journal of Ethnopharmacology, vol. 13, pp. 57-88. 
Park Young, Eun y Honn Jeon, 2008, "Equisetum hyemale, Antioxidant, Anti-inflammatory", en Natural Product Sciences, vol. 14, núm. 4, pp. 239-243.

Pérez Trejo, Gustavo, 1982, "La medicina”, en Esplendor del México antiguo, Centro de Investigaciones Antropológicas de México, Editorial del Valle de México, México.

Planta Medica, 1994, "Index of Biological and Pharmacological Effects", en Planta Medica, Journal of Medicinal Plant Research, vol. 60, núm. 6, serie XXXI.

_ 1995, "Index of Biological and Pharmacological Effects", en Planta Medica, Journal of Medicinal Plant Research, vol. 61, núm. 6, serie XXXIII.

Ritzer, George, 1993, "Sociología fenomenológica y etnometodológica”, en Teoría sociológica contemporánea, Mc Graw-Hill, México, pp. 263-297.

Rodríguez Ortiz, Imelda et al., 1989, "La medicina tradicional en el contexto de la filosofía de la cultura”, en Memorias del Tercer Coloquio de Medicina Tradicional Mexicana "Un Saber en Discusión", Escuela Nacional de Estudios Superiores Zaragoza, Universidad Nacional Autónoma de México, pp. 59-64.

Rodríguez-Ramos, Fernando y Andrés Navarrete, 2009, "Solving the Confusion of Gnaphaliin Structure: Gnaphaliin A and Gnaphaliin B Identified as Active Principles of Gnaphalium liebmanniiwith Tracheal Smooth Muscle Relaxant Properties", en Journal of Natural Products, vol. 72, núm. 6, pp. 1061-1064.

Rojas Gabriela, Juan Lévaro, Jaime Tortoriello y Víctor Navarro, 2001, "Antimicrobial Evaluation of Certain Plants Used in Mexican Traditional Medicine for the Treatment of Respiratory Diseases", en Journal of Ethnopharmacology, vol. 74, núm. 1, pp. 97-101.

Ryesky, Diana, 1976, Conceptos tradicionales de la medicina en un pueblo mexicano. Un análisis antropológico, Dirección General de Divulgación, Secretaría de Educación Pública, México.

Secretaría de Salud de Jalisco (ssı), Gobierno de Jalisco, 2009, en línea: <http://www.docstoc.com/docs/1130 36618/INFRELEVANTE-SALUD-JALISCO-PRESENTACI\% EF\%BF\%BDN-CARTA-2009>.
Speisky, Hernán y Bruce Cassels, 1994, "Boldo and Boldine: an Emerging Case of Natural Drug Development”, en Pharmacological Research, vol. 29, núm. 1, pp. 1-12.

Valadez, Isabel, Noé Alfaro y Elizabeth Pozos, 2003, "Cuidados alternativos en la atención del embarazo en Jalisco", en Cadernos de Saúde Pública, Río de Janeiro, vol. 19, núm. 5, pp. 1313-1321.

Vaverkova, Stefánia, Magda Holla y Joseph Tekel, 1995, "The Effect of Herbicides on the Qualitative Properties of the Healing Plants. Part 2: Content and Composition of the Essential Oil From Salvia Officinalis L. after Application of Afalon 50 WP", en Pharmazie, vol. 50, núm. 2, pp. 143-144.

Viesca Treviño, Carlos, 1992, "Veinte años de investigación en medicina tradicional de México", en Roberto Campos (comp.), La antropología médica en México, $\mathrm{t}$. II, Instituto de Investigaciones Históricas Dr. José María Luis Mora, Universidad Autónoma Metropolitana, México, pp. 115-138.

_ 1993 , "La herbolaria medicinal en México prehispánico”, en Jesús Kumate (comp.), La investigación científica de la herbolaria medicinal mexicana, Secretaría de Salud, México.

Viola, Haydeé et al., 1994, "Isolation of Pharmacologically Active Benzodiazepine Receptor Ligands from Tilia tomentosa (Tiliaceae)", en Journal of Ethnopharmacology, vol. 44, núm. 1, pp. 47-53.

Weller, Susan y Antone Kimball Romney, 1988, Systematic Data Collection, Sage Publications (Qualitative Research Methods Series, vol. 10), Londres.

Yeh, Gloria et al., 2003, "Systematic Review of Herbs and Dietary Supplements for Glycemic Control in Diabetes", en Diabetes Care, vol. 26, pp. 1277-1294.

Young-Jung, Lee et al., 2011, "Therapeutic Applications of Compounds in the Magnolia family", en Pharmacology \& Therapeutics, en línea: <http://www.sciencedirect.com/science/article/pii/S0163725811000349>. 